\title{
ÁCIDOS GRAXOS ESSENCIAIS E GERIOOX NO TRATAMENTO DO OLHO SECO INDUZIDO POR CIRURGIA DA CATARATA EM CÃES
}

\author{
Essential fatty acids and gerioox in treatment of dogs presenting \\ dry eye induced by cataract surgery
}

\begin{abstract}
Izabel Carolina Raittz Cavallet ${ }^{[a]}$, Antônio Felipe Paulino de Figueiredo Wouk ${ }^{[\mathrm{b}]}$
[a] Médica veterinária, Mestre em Ciências Veterinárias pela Universidade Federal do Paraná (UFPR), Professora colaboradora da Faculdade Estadual de Filosofia, Ciências e Letras de Paranaguá, Curitiba, PR - Brasil, e-mail: izabelcrc@yahoo.com.br

${ }^{[b]}$ Médico veterinário, professor titular da disciplina de Oftalmologia Veterinária da Universidade Federal do Paraná (UFPR), Curitiba, PR - Brasil, e-mail: fwouk@ufpr.br
\end{abstract}

\begin{abstract}
Resumo
Nesta pesquisa, objetivou-se identificar a ação de duas formulações de ômega 3 via oral em cães, apresentando epiteliopatia neurotrófica induzida por facectomia. Fizeram parte do estudo quatorze animais da raça Cocker Spaniel, portadores de luxação ou subluxação anterior bilateral do cristalino. Os animais foram divididos em grupos de sete pacientes para cada formulação, sendo que a primeira consistiu de ômega 3 de óleo de peixe e a segunda consistiu de ômega 3 associado a outros elementos (Gerioox). Os tratamentos tiveram início no quinto dia após a cirurgia e foram mantidos por sessenta dias. Foram realizadas avaliações oftálmicas no pré-operatório imediato e em seis diferentes momentos do pós-operatório. Além da oftalmoscopia direta, foi utilizado o teste lacrimal de Schirmer e o tempo de ruptura do filme lacrimal. No decorrer do tratamento, observou-se melhora do quadro clínico e recuperação em todos os animais. Houve evolução diferenciada dos parâmetros de qualidade e quantidade de lágrima entre os grupos. Uma melhora significativa $(\mathrm{p}<0,05)$ nos parâmetros do segundo grupo foi verificada, sendo o tempo de recuperação deste grupo menor. Como a formulação que apresentou melhor quadro evolutivo (Gerioox) possui menores quantidades de ômega 3, substância que tem indicação reconhecida no tratamento de olho seco, evidencia-se a necessidade de novas investigações desta formulação em particular e de seus demais componentes isoladamente, além de pesquisas sobre as diferentes fontes e dosagens de ômega 3 para o tratamento do olho seco em cães.
\end{abstract}

Palavras-chave: Facectomia. Olho seco. Filme lacrimal. Ômega 3. Antioxidantes. 


\begin{abstract}
The aim of the present investigation was to identify the action of two oral formulations of omega3 for dogs displaying neuroptrophic eptheliopathy induced by cataract surgery. A total of fourteen animals (Cocker Spaniel Breed) with bilateral anterior dislocation or subluxation of the lens were studied. The animals were divided in two groups of seven subjects for each of the treatments. The first formulation consisted of fish oil omega-3. The second formulation consisted of omega3 associated with other elements (Gerioox). The treatments began on the fifth day after surgery and were maintained for sixty days. Ophthalmic evaluations were performed immediately before surgery and in six different times of post-operative. Besides the direct ophthalmoscopy were used the Schirmer tear test 1 and the tear film break up test with fluorescein. In the development of the treatment, there was improvement in clinical status and recovery in all animals. There were different trends on the parameters of quality and quantity of tear between the two groups. A statistically significant improvement in the parameters and time for recovery of the group that received the second formulation was verified. As the formulation that showed better evolutionary framework contains smaller quantities of omega-3 (Gerioox), substance that has recognized indication for the treatment of dry eye, highlighting the need for further investigations in this particular formulation and its other components alone, in addition to research on the different sources and doses of omega-3 for treatment of dry eye in dogs.
\end{abstract}

Keywords: Cataract surgery. Dry eye. Tear film. Omega-3. Antioxidants.

\title{
INTRODUÇÃO
}

O emprego de ácidos graxos ômega 3 é bastante difundido, tanto na Medicina quanto na Medicina Veterinária, em medicações dermatológicas tópicas (como promotor da cicatrização) e como nutracêutico com diferentes indicações terapêuticas. Recentemente, muitos dos seus efeitos, até então pouco conhecidos, foram comprovados e entre estes estão incluídos os efeitos sobre o filme lacrimal (ESCAMILLA, 2003; HODGE et al., 2006; MILJANOVIC et al., 2005).

Sabe-se que, particularmente no olho humano, os ácidos graxos ômega 3 possuem efeitos imunossupressivos e anti-inflamatórios, que melhoram a expressão e a adesão dos microvilos dos epitélios oculares. Esta ação promove uma maior fluidez das secreções oriundas das glândulas tarsais, atuam no funcionamento das células caliciformes da conjuntiva e, assim, melhoram globalmente a qualidade do filme lacrimal e evitam uma rápida evaporação da lágrima (ESCAMILLA, 2003; PINHEIRO Jr. et al., 2007). O uso dos ácidos graxos essenciais em apresentação oral, na forma de óleo de linhaça ou óleo de peixe, é uma alternativa para o tratamento de diversas formas de deficiência lacrimal, embora isso seja pouco evidenciado na literatura (PINHEIRO Jr. et al., 2007).

O quadro de olho seco após a cirurgia de catarata é uma constante no homem, ocorrendo principalmente quando a técnica de facectomia extracapsular ou intracapsular é utilizada. Nestas técnicas, uma extensão maior da córnea é incisada, pois é necessária uma grande abertura na cápsula anterior do cristalino para se extrair o núcleo ou a lente em sua totalidade, lesando o nervo corneano, o que impede a lacrimação reflexa. A inflamação que ocorre por causa disso prejudica a produção e estabilidade lacrimal, induzindo uma epiteliopatia neurotrófica (ROBERTS; ELIE, 2007).

Pode-se supor que a epiteliopatia neurotrófica instalada após a facectomia seja decorrente da transecção de um número significativo de nervos sensoriais aferentes do plexo subepitelial durante a ablação do estroma corneano, o que ocasiona a diminuição da sensibilidade e interrupção do arcoreflexo que estimula tanto a secreção lacrimal basal quanto a reflexa (CREDIE et al., 2007; DAVIDSON; KUONEN, 2004; McCULLEY et al., 2006; WILSON, 2001). Em consequência da epiteliopatia neurotrófica induzida, ocorre uma instabilidade do filme lacrimal, gerando um círculo vicioso inflamatório 
(BREWITT; SISTANI, 2001; FRIDMAN et al., 2004). Além disso, informam ainda que as suturas e os colírios produzem a ruptura iatrogênica do filme lacrimal, ocasionando respostas imuno-inflamatórias que agravam a condição da Síndrome do Olho Seco.

A anestesia também pode causar diminuição da porção aquosa do filme lacrimal em humanos e animais. Em cães foram registradas significativas diminuições na produção lacrimal por mais de vinte e quatro horas após o evento anestésico ter sido terminado (CULLEN et al., 2005).

Apesar de existir técnica menos invasiva para o tratamento da catarata, a facoemulsificação, a facectomia extracapsular segue sendo a técnica mais empregada, principalmente na Oftalmologia Veterinária. Isso ocorre, sobretudo, por questões econômicas.

Por estas razões torna-se relevante o estudo de alternativas para o tratamento dos efeitos secundários da facectomia extracapsular ou intracapsular. Diante deste quadro, decidiu-se estudar a Síndrome do Olho Seco em cães após facectomia intra ou extracapsular por luxação ou subluxação anterior do cristalino, do ponto de vista de um novo paradigma terapêutico, que é o emprego dos ácidos graxos essenciais ômega 3, associados ou não a antioxidantes e oligoproteínas (Gerioox). Para este fim, foram usados cães de meia-idade predispostos ao olho seco e submetidos à facectomia intracapsular ou extracapsular por luxação ou subluxação anterior do cristalino.

\section{MATERIAL E MÉTODOS}

Ao longo de quatorze meses, foram selecionados da casuística de dois serviços universitários de Oftalmologia Veterinária - Universidade Federal do Paraná (UFPR) e Pontifícia Universidade Católica do Paraná (PUCPR) -, quatorze cães de meia idade, da raça Cocker Spaniel, portadores de subluxação ou luxação anterior bilateral do cristalino, com ou sem catarata, submetidos à facectomia bilateral intracapsular ou extracapsular. Todos os procedimentos foram realizados pelo mesmo cirurgião. Após avaliação clínica, três dias antes do procedimento cirúrgico, foi iniciada a medicação antibiótica tópica ocular com tobramicina, com seis instilações diárias. Duas horas antes do procedimento, a fim de manter a midríase per-operatória, foi administrado via oral $5 \mathrm{mg}$ de firocoxib (Previcox) por quilo, um novo anti-inflamatório não esteroidal (anti-prostaglandínico), inibidor seletivo de ciclooxigenase-2. Uma hora antes da intervenção, foram instilados colírios de sulfato de atropina a 1\% e cloridrato de fenilefrina a 10\%, a cada 15 minutos, para provocar midríase pré-operatória. Para a realização da anestesia geral, o paciente foi induzido com propofol e mantido com uma mistura de oxigênio e anestésico volátil. Foi também realizada uma anestesia local extra conal (peri-bulbar) com bupicavaína. Após a realização da antissepsia do fundo de saco conjuntival e a fixação do bulbo ocular, o início da facectomia ocorreu com uma incisão córneo-corneana límbica justa de 120 graus. Sob proteção do endotélio corneano com metilcelulose, realizou-se a capsulorrexe, expressão do núcleo e remoção de massas. As suturas da córnea foram realizadas com fio de poliglactina 8-0 com pontos isolados simples, sepultando-se o nó no estroma.

No pós-operatório, os animais seguiram com a mesma medicação e posologia antibiótica do pré-operatório por mais quinze dias, assim como com colírio de atropina a 1\%, três vezes ao dia por cinco dias. Foi empregado ainda um colírio de prednisona, três vezes ao dia por trinta dias, após o procedimento.

Os animais foram divididos ao acaso em dois grupos de sete pacientes. Cada grupo recebeu um tratamento nutracêutico diferenciado. Os animais do primeiro grupo, denominado grupo OMG (ômega 3), foram tratados com três cápsulas de ômega 3 de óleo de peixe que contêm em cada cápsula $87 \mathrm{mg}$ de EPA e $54 \mathrm{mg}$ de DHA (total: $261 \mathrm{mg}$ de EPA - ácido eicopentaenóico - e $162 \mathrm{mg}$ de DHA - ácido docosa-hexaenoico) por dia. Os demais animais formaram um segundo grupo, denominado grupo $G$ (Gerioox-Labyes), e receberam tratamento com um comprimido contendo $14 \mathrm{mg}$ de EPA e $21 \mathrm{mg}$ de DHA de origem tanto animal quanto vegetal, além de glucosamina destrogera $(0,140 \mathrm{~g})$, condroitin sulfato A $(0,15 \mathrm{~g})$, gluconato de cobre $(0,003 \mathrm{~g})$, gluconato de zinco $(0,02 \mathrm{~g})$, selenito de sódio $(0,05 \mathrm{~g})$ e vitamina $\mathrm{E}(1,8 \mathrm{~g})$. Esta formulação (Gerioox) é um novo medicamento indicado inicialmente como medicação geriátrica com efeitos antioxidantes, condroprotetores e dermatológicos para cães e gatos. Previamente ao início da 
pesquisa, os proprietários dos animais pertencentes ao grupo Gerioox $(G)$ foram alertados que seus animais estavam recebendo um tratamento alternativo ainda em teste. Somente após o consentimento e esclarecimento dos proprietários, os animais foram incluídos na pesquisa.

Em cada um dos grupos, a medicação foi administrada duas vezes ao dia, pela manhã e à noite, após a alimentação. Nenhum dos animais utilizados no estudo recebia ração ou outro tipo de suplementação contendo ômega 3 ou outro nutracêutico.

Os tratamentos dos dois grupos foram iniciados em todos os animais a partir do quinto dia de pós-operatório e foram mantidos durante um período de 60 dias.

A avaliação oftálmica e do filme lacrimal foi feita no período pré-operatório imediato (T0) e no quinto (T5), décimo (T10), décimo quinto (T15), trigésimo (T30), quadragésimo quinto (T45), sexagésimo (T60) e nonagésimo (T90) dias de pós-operatório. Para este fim, empregou-se oftalmoscópio direto, Teste Lacrimal de Schirmer 1 (STT1) e Tempo de ruptura do filme lacrimal (TRFL) com colírio de fluoresceína.

A metodologia usada para a análise foi a da comparação das médias de STT1 e TRFL entre os dois grupos experimentais realizada inicialmente por Análise de Variância (ANOVA). Os testes estatísticos de significância utilizados para comparação de médias foi o teste Tukey, sempre que o teste de Fisher da ANOVA constatou haver diferenças significativas no nível de probabilidade de 99\% e o uso do teste t de Student na comparação de dois processos, também no nível de $99 \%$.

\section{RESULTADOS E DISCUSSÃO}

Os procedimentos cirúrgicos ocorreram sem intercorrências. Não houve miose per-operatória e a midríase de $6 \mathrm{~mm}$ ou mais, obtida com a rotina de dilatação que incluiu a exposição dos animais ao firocoxib, atropina e fenilefrina permitiu a realização segura da facectomia intracapsular ou extracapsular em todos os cães.

O quadro de olho seco, caracterizado como uma doença inflamatória crônica, que apresenta anormalidades quantitativas e/ou qualitativas no filme lacrimal é frequente em cães. Os cães da raça Cocker Spaniel (particularmente fêmeas e animais de meia-idade) são predispostos, tanto à luxação do cristalino quanto ao olho seco senil (BREWITT; SISTANE, 2001; CABRAL et al., 2005; FRIDMAN et al., 2004).

O epitélio corneano contém numerosas terminações nervosas livres, o que explica a grande sensibilidade da córnea. A epiteliopatia corneana neurotrófica é atribuída à diminuição da produção lacrimal associada ao corte de nervos corneanos e influencia na regulação das secreções lacrimais, basal e reflexa (WILSON, 2001). A epiteliopatia neurotrófica e o consequente desenvolvimento do olho seco, derivados da cirurgia de facectomia intra ou extracapsular com grandes incisões corneanas, já foram descritos no homem por Roberts e Elie (2007) e, neste estudo, este fato se confirma em cães pela avaliação dos testes de quantidade (STT1) e estabilidade (TRFL) da lágrima quando comparados os dados do pré-operatório ( $\mathrm{T} 0$ ) com os dados obtidos após cinco dias do procedimento cirúrgico (T5).

Nas Figuras 1 e 2, são apresentadas as comparações entre as médias dos valores dos testes lacrimais quantitativo (STT1) e qualitativo (TRFL) nos dois grupos de tratamento durante todo o período do experimento. Quando comparados T0 e T5, os valores apresentados pelos testes reduziram drasticamente em todos os animais, caracterizando a instalação do quadro crítico de olho seco, sob os pontos de vista de qualidade (TRFL) e quantidade de lágrima (STT1). Estas alterações do filme lacrimal, provavelmente se instalaram por diferentes fatores: predisposição de raça e de idade; instalação de uma epiteliopatia neurotrófica e consequente diminuição da sensibilidade corneana e da produção lacrimal reflexa (DEWS, 2007; FRIDMAN et al., 2004); processos inflamatórios decorrentes de suturas e efeitos colaterais da anestesia (DEWS, 2007; MILJANOVIC et al., 2005). 
Ácidos graxos essenciais e gerioox no tratamento do olho seco induzido por cirurgia da catarata em cães

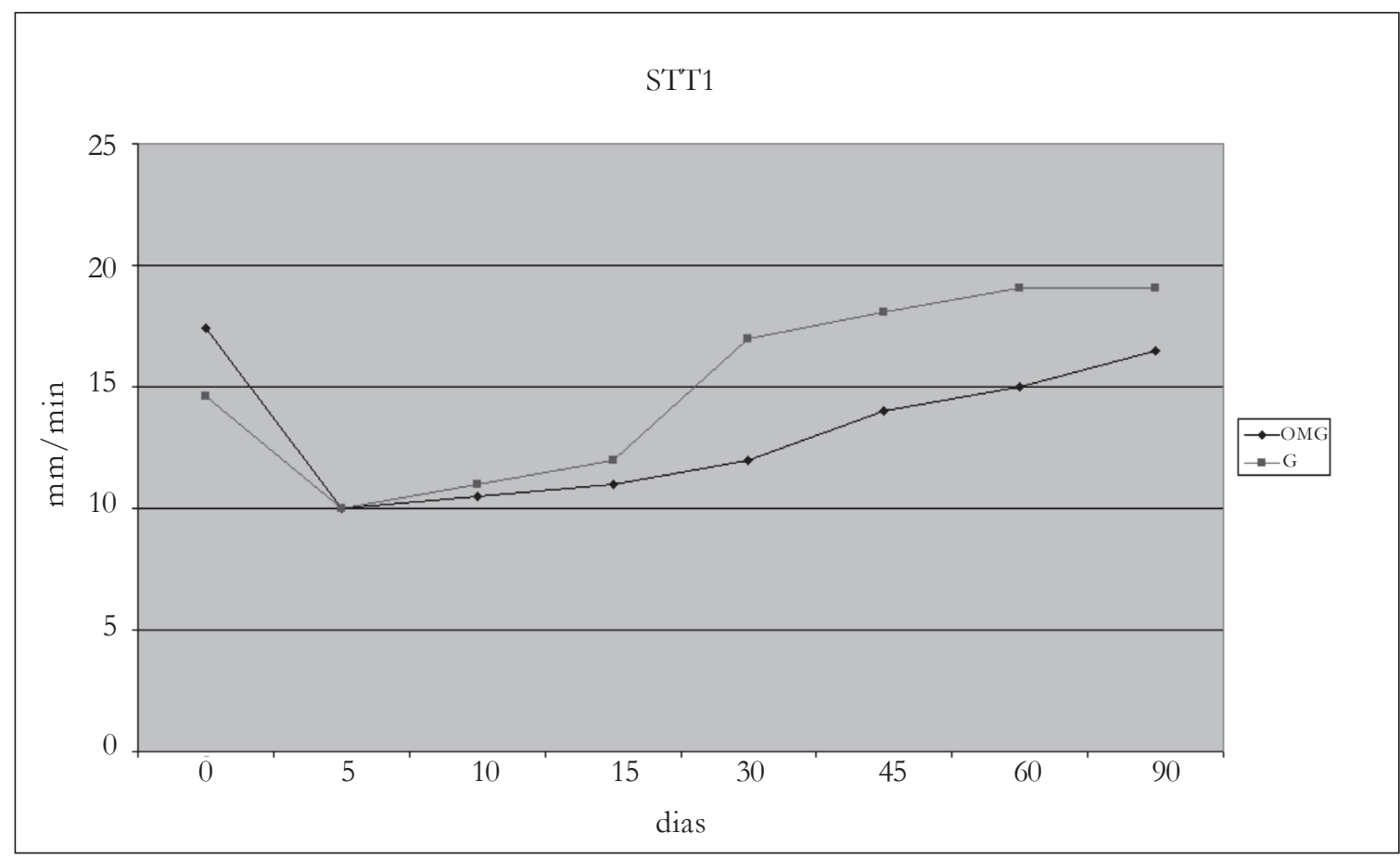

FIGURA 1 - Comparação das médias dos testes lacrimais quantitativos (stt1) entre os grupos de cães nos diferentes períodos. Curitiba, $2007(\mathrm{~N}=14)$

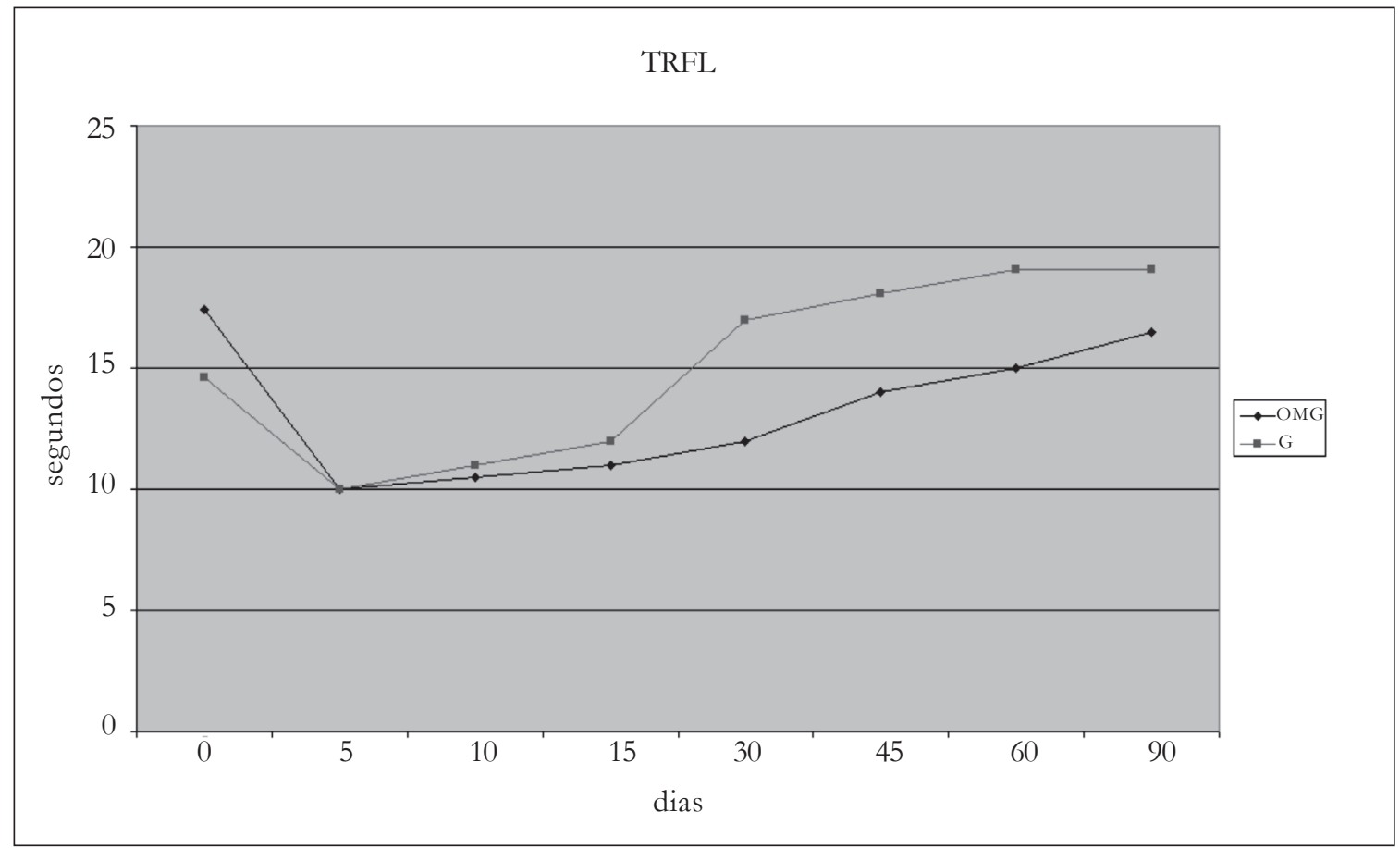

FIGURA 2 - Comparação das médias dos testes lacrimais qualitativos (trfl) entre os grupos de cães nos diferentes períodos. Curitiba, 2007 ( $\mathrm{N}=14)$ 
A doença do olho seco é acompanhada por vários graus de inflamação da superfície ocular. Evidencia-se um ciclo vicioso: a inflamação contribui para o olho seco e o olho seco ocasiona respostas imuno-inflamatórias agravando a própria situação de olho seco (DEWS, 2007; MILJANOVIC et al., 2005).

É conhecido que a recuperação da epiteliopatia neurotrófica induzida, em alguns casos, ocorre sem tratamento, em decorrência apenas da ação do sistema imune do próprio animal. Porém, isto pode levar meses de dor e desconforto e até acarretar problemas secundários maiores.

Vários tratamentos têm sido sugeridos para o olho seco, variando com a etiologia, gravidade e condições individuais de cada caso. Dentro do paradigma terapêutico pela via nutricional, escolhido para este estudo, o uso de ômega 3 e, mais recentemente, de sua associação com outros antioxidantes vem sendo discutido na comunidade científica, que tem observado sua eficácia no tratamento do olho seco tanto em humanos quanto em animais, ainda que não tenham chegado a conclusões específicas sobre as melhores fontes e associações destes (ESCAMILLA, 2003; HODGE et al., 2006; MILJANOVIC et al., 2005).

Levando em conta este estágio das pesquisas sobre o tema, neste estudo clínico foram testados, como tratamento, dois nutracêuticos existentes no mercado, um contendo apenas ácidos graxos ômega 3 e o outro contendo, além do ômega 3, outros componentes como vitaminas e minerais e glicosaminoglicanos.

Os dois grupos, quando comparados entre si em T0 e T5, não apresentaram diferenças estatisticamente significantes, desenvolvendo de uma maneira homogênea o quadro de olho seco e apresentando resultados clínicos e estatísticos semelhantes até o início do tratamento de ambos os grupos, no quinto dia de pós-operatório. Pode-se observar nos gráficos das Figuras 1 e 2 que houve grande queda nos valores dos testes após a cirurgia, quando comparados os valores em T0 e os valores em T5, evidenciando a instalação da epiteliopatia neurotrófica. Nos períodos posteriores (T10 a T90), observou-se um gradual aumento dos valores dos testes, sendo que ficam evidente as diferenças entre as curvas dos dois grupos, tanto para um teste quanto para outro, com os valores do grupo G sempre superiores e chegando com maior rapidez à normalidade da produção, estabilidade e qualidade do filme lacrimal. Quando avaliadas as evoluções dos quadros clínicos dos animais durante o tratamento como um todo em ambos os grupos, observou-se clinicamente nos animais do grupo G rápida melhora, equivalente a quase um terço do tempo levado pelo grupo OMG.

Quando comparada à soma das médias de todos os valores dos grupos OMG e G entre T5 e T90, as médias do grupo G mostraram-se superiores, sendo a diferença estatisticamente significante tanto para o TRFL quanto para o STT1. Analisando os períodos avaliados separadamente, usando o teste t de Student com probabilidade de 99\%, percebe-se que em T10 e T15 os grupos OMG e G respondem aos tratamentos de maneira bastante similar, o que pode ser notado tanto na observação dos gráficos das médias dos valores dos testes em cada grupo quanto estatisticamente.

A partir de T15, o tratamento atribuído ao grupo $G$ se mostra mais efetivo tanto clínica quanto estatisticamente. A maior diferença, estatisticamente significante, ocorre no período T30, ou seja, 25 dias após o início do tratamento. A partir de então, todos os valores analisados tanto para TRFL quanto para STT1 são superiores no grupo G. Nos períodos T45, T60 e T90, o grupo G continua apresentando resultados de qualidade e quantidade de lágrima superior, mas a significância estatística entre eles vai diminuindo.

O grupo OMG apresenta uma evolução do quadro mais lenta, embora constante, aproximando-se dos resultados do grupo $G$ em T90. O grupo $G$ apresenta um grande aumento nos valores dos testes entre T15 e T30 (10 a 25 dias de tratamento), quando então começa a estabilizar, não havendo diferença estatisticamente significante para o STT1 quando comparados em T30 e T45. Isto pode ser observado nas Figuras 1 e 2, onde a curva tende a se estabilizar a partir de T30, para ambos os testes, com maior destaque para o teste que avalia a produção lacrimal.

A literatura refere que $80 \%$ dos pacientes humanos com olho seco tratados com óleo de linhaça, outra fonte de ômega 3, têm a sua condição melhorada significativamente dois meses após o início do tratamento (PINHEIRO Jr. et al., 2007). Tal fato aqui se confirmou para o grupo OMG. No grupo $G$, uma grande melhora se deu entre 10 e 25 dias do uso do medicamento, com tendência à estabilidade e pequena evolução adicional ao longo do mês seguinte. 
A associação do ômega 3 a outros nutracêuticos pode ter se mostrado mais efetiva por causa dos efeitos antioxidativos e anti-inflamatórios dos componentes (WOUK et al., 2006) e também das interações deles entre si. Como exemplo dessas interações, pode-se citar o fato da vitamina E ter como uma de suas funções influenciar positivamente o metabolismo dos ácidos graxos ômega 3. Isto pode justificar o fato de que uma formulação contendo uma dose menor de ômega 3 tenha promovido resultados superiores em menor tempo de tratamento.

Outro aspecto importante a ser salientado é o fato de que no grupo $G$ a qualidade da lágrima entre T10 e T15 apresenta uma evolução superior à evolução da quantidade dela. Este pode ser um fator relevante para estudos posteriores e pode estar associado aos componentes nutracêuticos da fórmula, principalmente os antioxidantes contidos nela, pois se sabe que estes auxiliam na resposta antiinflamatória, na cicatrização corneana e mantêm a estabilidade do filme lacrimal (GUS et al., 2006).

Partindo-se do objetivo desta pesquisa de se estudar nutracêuticos como alternativas para o tratamento do olho seco secundário à técnica cirúrgica de facectomia, constatou-se que apesar de haver superioridade em uma das formulações utilizadas, ambas apresentaram eficácia e melhores resultados quando comparadas à evolução do quadro sem o uso de ácidos graxos ômega 3 vistos usualmente na prática médica veterinária. Como metabolicamente pode haver respostas diferenciadas às diferentes fontes de ômega 3, as pesquisas sobre estas devem ser continuadas, a fim de apontar eventuais diferenças, assim como aquelas pesquisas dirigidas ao estabelecimento da dosagem ideal e dos eventuais efeitos colaterais em tempos de estudo mais estendidos.

\section{CONCLUSÕES}

Nas condições deste estudo, comprovou-se a ocorrência de diminuição da quantidade e da qualidade da lágrima após facectomia em cães.

Os resultados evidenciaram que o firocoxib (Previcox) foi eficiente dentro das condições de uso neste estudo em relação à manutenção da dilatação pupilar, suficiência de midríase e ausência de miose per-operatória. Por esta razão, um estudo randomizado, com grupo placebo e mascarado, deverá ser realizado, a fim de se determinar a eficácia do firocoxib comparado a outras drogas quanto à manutenção per-operatória da midríase.

A terapia de suplementação de ácidos graxos essenciais (ômega 3 de óleo de peixe), assim como de outras drogas em associação com ácidos graxos essenciais (Gerioox), favorece a recuperação do olho seco induzido por facectomia em cães.

Forma terapêutica (Gerioox) revelou resultados superiores de Schirmer I e de Tempo de Ruptura do Filme Lacrimal durante o pós-operatório (particularmente no período de 30 a 45 dias).

\section{REFERÊNCIAS}

BREWITT, H.; SISTANI, F. Dry eye disease: the scale of problem. Survey of Ophthalmology, New York, v. 45, n. 2, p. 199-202, 2001.

CABRAL, V. P. et al. Canine lacrimal and third eyelid superficial glands macroscopic and morphometric characteristics. Ciência Rural, Santa Maria, v. 35, n. 2, p. 391-397, 2005.

CREDIE, M. G. et al. Alterações qualitativas do filme lacrimal após cirurgia refrativa: estudo comparativo entre PRK e LASIK. Arquivos Brasileiros de Oftalmologia, São Paulo, v. 70, n. 1, p. 23-30, 2007.

CULLEN, C. L.; LIM, C.; SYKEST, J. T. Tear film break up times in young healthy cats before and after anesthesia. Veterinary Ophthalmology, Chicago, n. 8, v. 3, p. 159-165, 2005. 
DAVIDSON, H. J.; KUONEN, V. J. The tear film and ocular mucins (Review). Veterinary Ophthalmology, Chicago, v. 7, n. 2, p. 71-77, 2004.

ESCAMILLA, N. E. R. Omega 3 y su acción terpéutica em el síndrome de ojo seco. Ciência y Tecnologia para la Salud Visual y Ocular, Bogotá, v. 1, n. 1, p. 91-98, 2003.

FRIDMAN, D. et al. Olho seco: conceitos, história natural e classificações. Arquivos Brasileiros de Oftalmologia, São Paulo, v. 67, n. 1, p. 181-185, 2004.

GUS, P. I. et al. Potencial antioxidativo da lágrima de adultos jovens. Arquivos Brasileiros de Oftalmologia, São Paulo, v. 69, n. 4, p. 565-570, 2006.

HODGE, W. G. et al. Efficacy of w-3 fatty acids in preventing age-related macular degeneration a systematic review. Ophthalmology, New York, v. 113, n. 7, p. 1165-1173, 2006.

INTERNATIONAL DRY EYE WORKSHOP - REPORT - DEWS. The epidemiology of dri eye disease. The Ocular Surface, Danvers, v. 5, n. 2, p. 93-107, 2007.

MILJANOVIC, B. et al. The relationship between dietary n-3 and n-6 fatty acids and clinically diagnosed dry eye syndrome in women. American Journal of Clinical Nutrition. Rockville Pike, v. 4, n. 82 , p. $887-893,2005$.

McCULLEY, J. P. et al. Impact of evaporation on aqueous tear loss. Transaction of the American Ophthalmological Society, San Francisco, v. 104, n. 8, p. 121-128, 2006.

PINHEIRO Jr., M. N. et al. Uso oral do óleo de linhaça (Linum Usitatissimum) no tratamento do olho seco de pacientes portadores da síndrome de sjogren. Arquivos Brasileiros de Oftalmologia, São Paulo, v. 4, n. 70, p. 649-655, 2007.

ROBERTS, C. W.; ELLIE, E. R. Dry eye symptons following cataract surgery. The Journal of the American Society of Ophthalmic Registered Nurses, San Francisco, v. 32, n. 1, p. 14-23, 2007.

WILSON, S. E. Laser in situ keratomielusis-induced (Presumed) neurotrophic epitheliopathy. Ophthalmology, New York, v. 108, n. 6, p. 1082-1087, 2001.

WOUK, A. F. P. F. et al. Ação do sulfato de condroitina "A" Associado a ciprofloxacina em úlceras de córnea de cavalos. Revista Acadêmica Ciências Agrárias e Ambientais, Curitiba, v. 4, n. 4, p. $11-20,2006$.

Recebido: 05/11/2008

Received: 11/05/2008

Aprovado: $20 / 03 / 2009$

Approved: 03/20/2009 